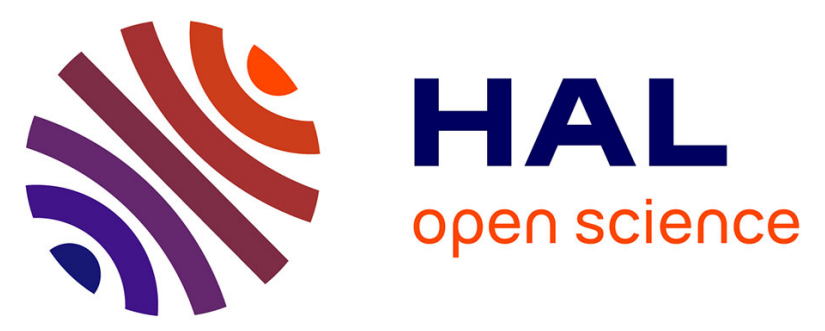

\title{
Characterization of the genome of human enteroviruses: design of generic primers for amplification and sequencing of different regions of the viral genome.
}

\author{
Maël Bessaud, Sophie Jegouic, Marie-Line Joffret, Christelle Barge, Jean \\ Balanant, Ionela Gouandjika-Vasilache, Francis Delpeyroux
}

\section{To cite this version:}

Maël Bessaud, Sophie Jegouic, Marie-Line Joffret, Christelle Barge, Jean Balanant, et al.. Characterization of the genome of human enteroviruses: design of generic primers for amplification and sequencing of different regions of the viral genome.. Journal of Virological Methods, 2008, 149 (2), pp.277-84. 10.1016/j.jviromet.2008.01.027 . pasteur-00318628

\section{HAL Id: pasteur-00318628}

https://hal-pasteur.archives-ouvertes.fr/pasteur-00318628

Submitted on 17 Sep 2008

HAL is a multi-disciplinary open access archive for the deposit and dissemination of scientific research documents, whether they are published or not. The documents may come from teaching and research institutions in France or abroad, or from public or private research centers.
L'archive ouverte pluridisciplinaire HAL, est destinée au dépôt et à la diffusion de documents scientifiques de niveau recherche, publiés ou non, émanant des établissements d'enseignement et de recherche français ou étrangers, des laboratoires publics ou privés. 
Characterization of the genome of human enteroviruses: design of generic primers for amplification and sequencing of different regions of the viral genome

Maël Bessaud $^{\mathrm{a}^{*}}$, Sophie Jegouic ${ }^{\mathrm{a}}$, Marie-Line Joffret ${ }^{\mathrm{a}}$, Christelle Barge ${ }^{\mathrm{a}, \mathrm{b}}$, Jean Balanant ${ }^{\mathrm{a}}$, Ionela Gouandjika-Vasilache ${ }^{\mathrm{c}}$, Francis Delpeyroux ${ }^{\mathrm{a}}$

${ }^{\text {a } U n i t e ́ ~ p o s t u l a n t e ~ d e ~ b i o l o g i e ~ d e s ~ v i r u s ~ e n t e ́ r i q u e s, ~ I n s t i t u t ~ P a s t e u r, ~} 25$ rue du Dr Roux, 75015

Paris, France

${ }^{\mathrm{b}}$ Centre de ressources biologiques de l'Institut Pasteur, Institut Pasteur, 25 rue du Dr Roux, 75015 Paris, France

${ }^{\mathrm{c}}$ Laboratoire des Entérovirus/Rougeole, Institut Pasteur de Bangui, BP 923, Bangui, République Centrafricaine

*Corresponding author. Present address: Unité postulante de biologie des virus entériques, Institut Pasteur, 25 rue du Dr Roux, 75015 Paris, France. Phone: +33 1406133 22. Fax: +33 1406133 67. e-mail: bessaud@pasteur.fr 


\begin{abstract}
Human enteroviruses are among the most common viruses infecting humans and can cause diverse clinical syndromes ranging from minor febrile illness to severe and potentially fatal diseases. Biodiversity and evolution of human enterovirus genomes are shaped by frequent recombination events. Therefore, identification and characterization of circulating strains of enteroviruses requires partial determination of different genomic regions.

This article reports the development of a simple method allowing amplification and partial sequencing of the P1, P2 and P3 genomic regions of field human enterovirus strains isolated in cell cultures, by performing PCR on cDNAs generated through a single RT reaction. A set of generic primers were designed and tested on a panel of 90 field and prototype viruses belonging to the five species of human enteroviruses. This assay was shown to amplify efficiently the targeted regions of all the 90 genomes tested. The generated amplicons were sequenced successfully without the need for gel purification.

This assay could be a precious tool for laboratories interested in molecular epidemiology and evolution studies implicating a great number of human enterovirus strains isolated from human or environmental samples.
\end{abstract}

Keywords: coxsackievirus, echovirus, genotyping, human enterovirus, poliovirus, random primer 


\section{Introduction}

Human enteroviruses, members of the genus Enterovirus, family Picornaviridae, are among the most common viruses infecting humans. Although most infections with human enteroviruses are asymptomatic or result in mild infection, human enteroviruses can cause diverse clinical syndromes ranging from minor febrile illness to severe and potentially fatal diseases, such as acute hemorrhagic conjunctivitis, aseptic meningo-encephalitis, and acute flaccid paralysis (Palacios and Oberste, 2005).

Enterovirus virions are non-enveloped and contain a single positive-sense RNA genome of about 7,500 nucleotides (nt) with only one open-reading frame (ORF) flanked by untranslated regions (Figure 1A). This ORF encodes a single polyprotein. Co- and posttranslational proteolytic processing gives rise to three precursors (P1-P3) secondarily cleaved in four structural proteins (VP1-VP4), which are assembled into the virion, and non-structural proteins, which are expressed in infected cells (Pallansch and Roos, 2001).

Historically, human enteroviruses have been classified into echoviruses (EV), coxsackievirus groups A and B (CV-A and CV-B), and polioviruses (PV), according to their phenotypic and pathogenic properties. Current taxonomy, based essentially on phylogenetic studies, divides human enteroviruses into 5 species (Hyypia et al., 1997): HEV-A (at least 17 serotypes), HEV-B (at least 56 serotypes), HEV-C (at least 13 serotypes), HEV-D (at least 3 serotypes) and the 3 serotypes of PV.

After isolation in cell culture, typing of human enteroviruses consists in identifying the serotype by seroneutralization. Because of their labour intensiveness, neutralization methods have been replaced by molecular tools in many laboratories. Indeed, a correlation between serotype and genotype for the P1 region of the genome has been demonstrated (Kottaridi et 
al., 2005; Oberste et al., 1999). Therefore, several methods have been developed in order to determine partly the sequences encoding the VP1 protein (Bailly et al., 2000; Caro et al., 2001; Casas et al., 2001; Iturriza-Gomara et al., 2006; Nix et al., 2006; Norder et al., 2001; Oberste et al., 2003; Thoelen et al., 2003), which plays a key role in the constitution of the viral neutralization antigenic sites. Methods targeting other capsid-encoding regions, such as VP2 or VP4, have also been developed (Ishiko et al., 2002; Nasri et al., 2007). These methods were shown to be suitable for the purpose of determining the serotypes of field or clinical strains. However, the determination of the serotype appears insufficient for surveillance or research purpose, particularly for studying the molecular epidemiology and evolution of human enteroviruses. Indeed, recombination events between enteroviruses are known to be very frequent (Dahourou et al., 2002; Lindberg et al., 2003; Lukashev, 2005; Oberste et al., 2004; Oprisan et al., 2002; Santti et al., 1999), leading to a wide range of mosaic genomes inside human enterovirus species (Chen et al., 2007; Lukashev et al., 2003; Oberste et al., 2004; Rakoto-Andrianarivelo et al., 2007; Simmonds and Welch, 2006). Recombination is now considered as an important way of evolution of human enteroviruses (Chevaliez et al., 2004; Lukashev, 2005; Oberste et al., 2004), which can give rise to new viral genotypes exhibiting modified pathogenic properties. Thus, recombinant viruses associating capsid sequences of vaccine-derived strains of PV and sequences from HEV-C have been rendered responsible for several outbreaks of acute poliomyelitis (Anonymous, 2001a; Anonymous, 2001b; Kew et al., 2004; Rousset et al., 2003). Moreover, recombination could explain the failure of attempts to correlate serotypes and clinical symptoms in most human enterovirusinduced diseases.

While recombination is observed in the capsid-encoding region (Blomqvist et al., 2003; Bouslama et al., 2007; Martin et al., 2002), recombination events occur mainly in the P2 and P3 regions of the genome (Arita et al., 2005; Cuervo et al., 2001; King, 1988; 
Simmonds and Welch, 2006). Therefore, typing approaches based solely on the capsid sequence appear to be insufficient for the survey of human enterovirus circulation and for the understanding of human enterovirus evolution, which require the determination of sequences of other genomic regions, particularly the non-structural protein-encoding sequences.

In order to make this determination easier, a simple method was developed, which allows amplification and partial sequencing of the genomic regions of each of the precursors $\mathrm{P} 1$ to $\mathrm{P} 3$ of field human enterovirus strains isolated in cell cultures, by performing PCR on cDNAs generated through a single RT reaction.

\section{Materials and methods}

\subsection{Virus isolates}

The prototype viruses were provided by the National Institute of Public Health and the Environment (RIVM) (Bilthoven, The Netherlands). The field isolates were from stools collected in Central African Republic and in Madagascar from 2001 to 2007. These isolates were typed by partial sequencing of their VP1-encoding gene following the method already described (Nix et al., 2006).

\subsection{Sequence analysis}

Human enteroviruses full-length polyprotein sequences retrieved from the GenBank database were used to design the primers. Viral amino-acidic conserved regions were identified by generating multiple alignment using CLC Combined Workbench 3.0 software (CLC bio, Aarhus, Denmark).

\subsection{Virus preparation and RNA extraction}


All work with infectious viruses was carried out in a BSL-2 facility. HEV-A and B were grown in RD cells, HEV-C and PV in HEp2c cells and HEV-D in GaBi cells (Kapsenberg, 1968).

Viral RNAs were extracted from $250 \mu \mathrm{L}$ of culture supernatants using the High Pure Viral RNA kit (Roche Diagnostics, Meylan, France), following the manufacturer's instructions.

\subsection{RT-PCR conditions}

Primers (Eurogentec, Liège, Belgium) are listed in Table 1. Working solutions were prepared at a concentration of $20 \mu \mathrm{M}$, except for heptaN, which was dissolved at $100 \mu \mathrm{g} . \mathrm{mL}^{-}$ 1.

cDNA synthesis was performed in a final volume of $10.5 \mu \mathrm{L}$ using the SuperScript II reverse transcriptase (Invitrogen, Cergy-Pontoise, France); the reaction mixture contained 5 $\mu \mathrm{L}$ of purified viral RNA, $2 \mu \mathrm{L}$ of $5 \mathrm{X}$ First-Strand Buffer, $0.01 \mathrm{M}$ dithiothreitol $(1 \mu \mathrm{L}), 100$ ng of the random primers heptaN $(1 \mu \mathrm{L}), 10$ pmol of each $\mathrm{dNTP}(1 \mu \mathrm{L}$ of a $10 \mathrm{mM}$ mixture $)$ and $100 \mathrm{U}$ of enzyme $(0.5 \mu \mathrm{L})$. The RT reaction mixture was then incubated at $25{ }^{\circ} \mathrm{C}$ for 10 $\min , 42^{\circ} \mathrm{C}$ for $45 \mathrm{~min}$ and $95^{\circ} \mathrm{C}$ for $5 \mathrm{~min}$.

cDNAs were then used as templates for amplification in PCR carried out in a final volume of $50 \mu \mathrm{L}$ that included $5 \mu \mathrm{L}$ of $10 \mathrm{X}$ PCR Buffer, $200 \mu \mathrm{M}$ of each dNTP, $50 \mathrm{pmol}(2.5$ $\mu \mathrm{L}$ ) of each primer, $2 \mu \mathrm{L}$ of cDNA and $2.5 \mathrm{U}$ of HotStartTaq DNA polymerase (Qiagen, Courtabœuf, France). The thermocycler profile was $15 \mathrm{~min}$ at $95{ }^{\circ} \mathrm{C}$ followed by 30 cycles of $30 \mathrm{~s}$ at $95^{\circ} \mathrm{C}, 30 \mathrm{~s}$ at $45^{\circ} \mathrm{C}$ and $2 \mathrm{~min}$ at $60^{\circ} \mathrm{C}$. For semi-nested PCR (snPCR), the reaction was achieved on $1 \mu \mathrm{L}$ of the first amplification using $1.25 \mathrm{U}$ of DNA polymerase under the same experimental procedures.

The PCR products were analysed on BET-stained agarose gels. 


\subsection{Sequencing of the amplicons}

PCR products were purified by silica-based purification (Qiaquick, Qiagen). The sequences of the resulting amplicons were checked by sequencing with a BigDye terminator v3.1 kit (Applied Biosystems, Courtabœuf, France) on an ABI Prism 3140 automated sequencer (Applied Biosystems).

The amplicons generated with tailed primers were sequenced using primers M13Fwd and M13Rev (Table 1). The other ones were sequenced using PCR primers.

\subsection{Assay sensitivity}

The sensitivity of the assay was measured by using supernatants of cell cultures infected by PV-1 strain Sabin and EV-7 strain Wallace. The titers of these supernatants, expressed as $\mathrm{TCID}_{50}$ per $\mathrm{mL}$, were determined according to the WHO standard protocol (Anonymous, 2004). Serial 4-fold dilutions of the supernatants were made in cell culture medium. RNA was extracted and subjected to RT and PCR as exposed in sections 2.3 and 2.4.

\section{Results}

\subsection{Sequence analysis and primer design}

In order to amplify successfully regions located in different parts of the genome of human enteroviruses, generic primers were designed to target genomic sequences that encode conserved peptidic motifs in the viral polyprotein (Figure 1B).

In the P1 region, two conserved amino acid sequences were identified on both sides VP1. The sense primer AMTH targeted a region located $~ 270$ nt upstream the VP1-encoding sequence. This region was also targeted by the primer 224 in a previously reported snPCR 
assay (Nix et al., 2006); nevertheless, compared to the Nix' primer, the primer AMTH was slightly modified to take into account particular amino acidic sequences featured by several viruses, such as EV-13, HEV-69 and viruses belonging to the HEV-D species. In the P2 region of the genome, the primers targeted sequences encoding conserved cleavage sites of the polyprotein, at the $2 \mathrm{~A}-2 \mathrm{~B}$ and $2 \mathrm{C}-3 \mathrm{~A}$ junctions. In the $\mathrm{P} 3$ region, the primers targeted highly conserved sequences in the $3 \mathrm{C}$ and $3 \mathrm{D}$ regions.

Consensus degenerate primers were designed by back-translating the conserved peptidic patterns into nucleotidic sequences. Deoxyinosine was introduced at the positions displaying 4-fold codon degeneracy. In order to simplify the sequencing step, the PCR primers were tagged with commonly-used M13 forward and reverse sequences to produce M13-labelled PCR products. Labelling of PCR products allowed the utilisation of identical primers for the sequencing of amplicons produced with different primer pairs (Bailly et al., 2000).

\subsection{Assay evaluation}

In a first time, the new primers pairs were tested on PV-1 and EV-7 supernatants whose titers was determined to be $10^{8.4}$ and $10^{8.0} \mathrm{TCID}_{50}$ per $\mathrm{mL}$, respectively. Following the RT step with random primers performed on viral RNA extracted from undiluted supernatants, the primer pairs J-AMTH/J-GDCL, J-HEV.2AB.d/J-HEV.2C3A.c and J-HEV.2C.d1/JHEV.3D.c2 produced detectable gel bands (Figure 2). The amplicons were successfully sequenced using M13 primers without gel extraction.

The same assay was then performed on PV-1 and EV-7 RNA extracted from diluted supernatants in order to determine the sensitivity of each primer pair (Figure 2 and Table 2). 
On PV-1 RNA, the P2-targeting pair J-HEV.2AB.d/J-HEV.2C3A.c was able to generate amplicons for supernatants displaying a titer $\geq 10^{5.4} \mathrm{TCID}_{50}$ per $\mathrm{mL}$ whereas $\mathrm{J}$ HEV.3C.d1/J-HEV.3D.c2 (P3 region) required titer $\geq 10^{6.0} \mathrm{TCID}_{50}$ per $\mathrm{mL}$. The P1-targeting pair J-AMTH/J-GDCL was found to be poorly sensitive, requiring a titer $\geq 10^{7.0} \mathrm{TCID}_{50}$ per $\mathrm{mL}$ for positive results.

In an attempt to improve the sensitivity of the PCR assays, reactions were performed using the same primers deprived of M13 tags. In the P1 region, the new primers (called AMTH and GDCL) improved the sensitivity, getting a positive result for supernatants with titer $\geq 10^{6.0} \mathrm{TCID}_{50}$ per $\mathrm{mL}$ (Figure 2). Therefore, only the untagged primer pairs AMTH/GDCL was used to amplify the P1 region of the genome in the subsequent experiments. In contrast, in the $\mathrm{P} 3$ and $\mathrm{P} 2$ regions of the genome, achievement of the PCR with untagged primer pairs (HEV.3C.d1/HEV.3D.c2 and HEV.2AB.d/HEV.2C3A.c, respectively) did not improve dramatically the sensitivity of the assay (Table 2): in the P3 region, the untagged primers led to a slight improvement of the PCR sensitivity $\left(10^{5.4}\right.$ instead of $\left.10^{6.0}\right)$; in the $\mathrm{P} 2$ region of the genome, no improvement was observed by using untagged primers.

In order to improve the sensitivity of the method, snPCR were assayed in the three regions of the genome. In the P1 region, the primers 224 and 222 previously designed by Nix et al. (Nix et al., 2006) were used; in the P3 region of the genome, a new primer ( $\mathrm{J}$ HEV.3C.d2) was designed. The P1- and P3-targeting primers were found to be able to generate detectable amplicons for supernatants featuring titer as low as $10^{3.0} \mathrm{TCID}_{50}$ per $\mathrm{mL}$ (Figure 2). In contrast, improvement of the sensitivity of the assay by snPCR in the P2 region was unsuccessful: all the primers designed in this region failed to achieve an amplification leading to gel bands suitable for direct sequencing (data not shown). 
Sensitivity assays achieved with the EV-7 supernatant gave similar sensitivity values (data not shown).

In a second step, the assay was tested on 90 randomly-chosen prototype strains and field isolates available in the laboratory. These strains belonged to 39 different serotypes representative of the five human enterovirus species (Table 3). The assay succeeded in amplifying the P1 and the P3 regions for all the 90 tested strains either by RT-PCR (62 isolates of 90) or by RT-snPCR. For most of them (65 isolates of 90), the P2 region was also amplified by RT-PCR. In all cases, the PCR and/or snPCR assays led to gel-bands that were efficiently sequenced without gel extraction step.

\section{Discussion}

Human infections with enteroviruses are very frequent. Recombination events between different human enteroviruses are known to be very common, giving rise to a wide range of genotypes, of which some can exhibit threatening pathogenic properties. For this reason, many laboratories are involved worldwide in the surveillance of the human enterovirus circulation, particularly in countries with insufficient vaccine coverage against poliomyelitis. The surveillance, based on isolation of field strains, implies not only the determination of the serotype of these viruses (either by seroneutralization or by sequencing the capsid-encoding region), but also the determination of sequences of other genomic regions, in particular those of the non-structural moiety. Large-scale sequencing of circulating human enteroviruses, both in their structural and non-structural regions, is expected to provide new insights to understand better enterovirus genetics and to evaluate the possibility of emergence of new genotypes with increased pathogenic power. 
The aim of this study was to develop a convenient method allowing the sequencing of regions distributed in the entire ORF of the genome of human enterovirus strains isolated in cell cultures. For this purpose, generic primers were designed by back-translating genomic sequences that encode peptidic patterns highly conserved among the five human enterovirus species. Some of the identified regions have already been targeted by other primers previously reported, such as CHR2, a primer targeting the 2C-3A junction of EV (Kottaridi et al., 2007), or the primers EUC12-a, -b and -c designed to partly sequence the 3D-coding region of HEVB (Oprisan et al., 2002). After back-translation, the linear increase of the primer degeneracy level can be detrimental to amplification sensitivity due to the geometric increase in combinatory possibilities (Rossolini et al., 1994); for that reason, deoxyinosine was introduced in the primers at the nucleotidic positions displaying 4-fold degeneration.

Compared to the pair AMTH/GDCL, the lack of sensitivity of the primer pair JAMTH/J-GDCL observed in the pilot experiments with PV-1 and EV-7 could be explained by the interference of the M13 tags, which could inhibit the reaction by steric hindrance or by creating fortuitous secondary structures. In contrast, untagged primers did not improve drastically the sensitivity of the P2- and P3-targeting assays. These results illustrate the fact that the influence of the primer tails on PCR yield is not clear, as already observed (Afonina et al., 2007).

For viral strains with titer $\geq 10^{6.0} \mathrm{TCID}_{50}$ per $\mathrm{mL}$, the three amplicons obtained after the first round of PCR represented $\sim 4,100 \mathrm{nt}$, i.e. more than $50 \%$ of the whole genomic sequence. In the P1 region of the genome, the AMTH/GDCL PCR product encompassed the full VP1 gene, providing more complete information than the previously reported pan-human enterovirus VP1-targeting assays (Caro et al., 2001; Nix et al., 2006), designed to identify the serotype of field or clinical strains. 
When grown in cell cultures, some human enteroviruses can display a titer lower than $10^{6.0} \mathrm{TCID}_{50}$ per $\mathrm{mL}$. To make possible the typing of such viruses, snPCR were developed to increase the sensitivity of the assays. snPCR in the $\mathrm{P} 1$ and $\mathrm{P} 3$ regions were shown to be able to amplify sequences of virus with titer as low as $10^{3.0} \mathrm{TCID}_{50}$ per $\mathrm{mL}$, giving rise to products covering about one quarter of the viral genome. In spite of the absence of snPCR in the P2 region, the majority of the tested strains grown in cell cultures were successfully amplified in this region by the single PCR with the primers J-HEV.2AB.d/J-HEV.2C3A.c.

Compared to other known methods developed to sequence genomic regions of human enteroviruses, this new assay has several advantages. First, contrary to other methods that require a specific primer set for each species (Iturriza-Gomara et al., 2006; Junttila et al., 2007), this assay succeeded in amplifying genome of field strains belonging to the five human enterovirus species. Second, the use of random primers for the RT reaction made it possible to reduce the workload, the same RT product serving as matrix in the three following PCR assays. Third, the purity of the amplicons and their sizes, lower than 1,500 bp, allowed the determination of overlapping sense and anti-sense sequences in a single round of sequencing without the need for gel-extraction.

In summary, a convenient method was developed for the determination of sequences of $\mathrm{P} 1, \mathrm{P} 2$ and $\mathrm{P} 3$ regions of the genome of human enteroviruses isolated in cell cultures. This method may be extremely useful for laboratories involved in the enterovirus survey, to type circulating viruses and to study the phenomenon of recombination between enteroviruses.

\section{Ackowledgements}


The authors are indebted to Laure Diancourt and Coralie Tran (Plateforme Santé publique, Institut Pasteur de Paris) for amplicon sequencing, to Alexandre Manirakiza, Arthur Mazitchi, Jean Fandema and Matthew Pflaum for excellent technical assistance and thank Florence Colbère-Garapin for manuscript revision and Javier Martin for skilful discussions.

S.J. is granted by the French Délégation générale pour l'armement and the French Centre national pour la recherche scientifique (CNRS). This work was funded by the Pasteur Institute: Direction des PTR (Projet transversal de recherche $n^{\circ} 276$ ) and Direction des affaires internationales (ACIP 06-2007 and ACIP 22-2006). 


\section{References}

Afonina, I., Ankoudinova, I., Mills, A., Lokhov, S., Huynh, P. and Mahoney, W., 2007. Primers with 5' flaps improve real-time PCR. Biotechniques 43, 770-773.

Anonymous, 2001a. Acute flaccid paralysis associated with circulating vaccine-derived poliovirus, Philippines, 2001. Wkly Epidemiol Rec 76, 319-20.

Anonymous, 2001b. Circulation of a type 2 vaccine-derived poliovirus, Egypt. Wkly Epidemiol Rec 76, 27-9.

Anonymous. 2004. Titration of laboratory quality control standard. In: WHO (Ed), Polio laboratory manual, World Health Organization, Geneva, pp. 75-78.

Arita, M., Zhu, S.L., Yoshida, H., Yoneyama, T., Miyamura, T. and Shimizu, H., 2005. A Sabin 3-derived poliovirus recombinant contained a sequence homologous with indigenous human enterovirus species $\mathrm{C}$ in the viral polymerase coding region. J Virol $79,12650-7$.

Bailly, J.L., Beguet, A., Chambon, M., Henquell, C. and Peigue-Lafeuille, H., 2000. Nosocomial transmission of echovirus 30: molecular evidence by phylogenetic analysis of the VP1 encoding sequence. J Clin Microbiol 38, 2889-92.

Blomqvist, S., Bruu, A.L., Stenvik, M. and Hovi, T., 2003. Characterization of a recombinant type $3 /$ type 2 poliovirus isolated from a healthy vaccinee and containing a chimeric capsid protein VP1. J Gen Virol 84, 573-80.

Bouslama, L., Nasri, D., Chollet, L., Belguith, K., Bourlet, T., Aouni, M., Pozzetto, B. and Pillet, S., 2007. Natural recombination event within the capsid genomic region leading to a chimeric strain of human enterovirus B. J Virol 81, 8944-52.

Caro, V., Guillot, S., Delpeyroux, F. and Crainic, R., 2001. Molecular strategy for 'serotyping' of human enteroviruses. J Gen Virol 82, 79-91. 
Casas, I., Palacios, G.F., Trallero, G., Cisterna, D., Freire, M.C. and Tenorio, A., 2001. Molecular characterization of human enteroviruses in clinical samples: comparison between VP2, VP1, and RNA polymerase regions using RT nested PCR assays and direct sequencing of products. J Med Virol 65, 138-48.

Chen, G.W., Huang, J.H., Lo, Y.L., Tsao, K.C. and Chang, S.C., 2007. Mosaic genome structure of echovirus type 30 that circulated in Taiwan in 2001. Arch Virol 152, 1807-17.

Chevaliez, S., Szendroi, A., Caro, V., Balanant, J., Guillot, S., Berencsi, G. and Delpeyroux, F., 2004. Molecular comparison of echovirus 11 strains circulating in Europe during an epidemic of multisystem hemorrhagic disease of infants indicates that evolution generally occurs by recombination. Virology $325,56-70$.

Cuervo, N., Guillot, S., Romanenkova, N., Combiescu, M., Aubert-Combiescu, A., Seghier, M., Caro, V., Guillot, S., Crainic, R. and Delpeyroux, F., 2001. Genomic features of intertypic recombinant Sabin strains excreted by primary vaccinees. J. Virol. 75, $5740-5751$.

Dahourou, G., Guillot, S., Le Gall, O. and Crainic, R., 2002. Genetic recombination in wildtype poliovirus. J Gen Virol 83, 3103-10.

Hyypia, T., Hovi, T., Knowles, N.J. and Stanway, G., 1997. Classification of enteroviruses based on molecular and biological properties. J Gen Virol 78 ( Pt 1), 1-11.

Ishiko, H., Shimada, Y., Yonaha, M., Hashimoto, O., Hayashi, A., Sakae, K. and Takeda, N., 2002. Molecular diagnosis of human enteroviruses by phylogeny-based classification by use of the VP4 sequence. J Infect Dis 185, 744-54.

Iturriza-Gomara, M., Megson, B. and Gray, J., 2006. Molecular detection and characterization of human enteroviruses directly from clinical samples using RT-PCR and DNA sequencing. J Med Virol 78, 243-53. 
Junttila, N., Leveque, N., Kabue, J.P., Cartet, G., Mushiya, F., Muyembe-Tamfum, J.J., Trompette, A., Lina, B., Magnius, L.O., Chomel, J.J. and Norder, H., 2007. New enteroviruses, EV-93 and EV-94, associated with acute flaccid paralysis in the Democratic Republic of the Congo. J Med Virol 79, 393-400.

Kapsenberg, J.G., 1968. ECHO virus type 33 as a cause of meningitis. Arch Gesamte Virusforsch 23, 144-7.

Kew, O.M., Wright, P.F., Agol, V.I., Delpeyroux, F., Shimizu, H., Nathanson, N. and Pallansch, M.A., 2004. Circulating vaccine-derived polioviruses: current state of knowledge. Bull World Health Organ 82, 16-23.

King, A.M., 1988. Preferred sites of recombination in poliovirus RNA: an analysis of 40 intertypic cross-over sequences. Nucleic Acids Res 16, 11705-23.

Kottaridi, C., Bolanaki, E., Kyriakopoulou, Z., Dedepsidis, E., Pratti, A. and Markoulatos, P., 2007. Possible recombination and gene adaptation exchanges among clinical echovirus strains: crossing the temporal and topological barriers. Diagn Microbiol Infect Dis 58, 407-12.

Kottaridi, C., Bolanaki, E., Siafakas, N. and Markoulatos, P., 2005. Evaluation of seroneutralization and molecular diagnostic methods for echovirus identification. Diagn Microbiol Infect Dis 53, 113-9.

Lindberg, A.M., Andersson, P., Savolainen, C., Mulders, M.N. and Hovi, T., 2003. Evolution of the genome of Human enterovirus B: incongruence between phylogenies of the VP1 and 3CD regions indicates frequent recombination within the species. J Gen Virol $84,1223-35$.

Lukashev, A.N., 2005. Role of recombination in evolution of enteroviruses. Rev Med Virol $15,157-67$. 
Lukashev, A.N., Lashkevich, V.A., Ivanova, O.E., Koroleva, G.A., Hinkkanen, A.E. and Ilonen, J., 2003. Recombination in circulating enteroviruses. J Virol 77, 10423-31.

Martin, J., Samoilovich, E., Dunn, G., Lackenby, A., Feldman, E., Heath, A., Svirchevskaya, E., Cooper, G., Yermalovich, M. and Minor, P.D., 2002. Isolation of an intertypic poliovirus capsid recombinant from a child with vaccine-associated paralytic poliomyelitis. J Virol 76, 10921-8.

Nasri, D., Bouslama, L., Omar, S., Saoudin, H., Bourlet, T., Aouni, M., Pozzetto, B. and Pillet, S., 2007. Typing of human enterovirus by partial sequencing of VP2. J Clin Microbiol 45, 2370-9.

Nix, W.A., Oberste, M.S. and Pallansch, M.A., 2006. Sensitive, seminested PCR amplification of VP1 sequences for direct identification of all enterovirus serotypes from original clinical specimens. J Clin Microbiol 44, 2698-704.

Norder, H., Bjerregaard, L. and Magnius, L.O., 2001. Homotypic echoviruses share aminoterminal VP1 sequence homology applicable for typing. J Med Virol 63, 35-44.

Oberste, M.S., Maher, K., Kilpatrick, D.R. and Pallansch, M.A., 1999. Molecular evolution of the human enteroviruses: correlation of serotype with VP1 sequence and application to picornavirus classification. J. Virol. 73, 1941-1948.

Oberste, M.S., Maher, K. and Pallansch, M.A., 2004. Evidence for frequent recombination within species human enterovirus B based on complete genomic sequences of all thirty-seven serotypes. J Virol 78, 855-67.

Oberste, M.S., Nix, W.A., Maher, K. and Pallansch, M.A., 2003. Improved molecular identification of enteroviruses by RT-PCR and amplicon sequencing. J Clin Virol 26, $375-7$. 
Oprisan, G., Combiescu, M., Guillot, S., Caro, V., Combiescu, A., Delpeyroux, F. and Crainic, R., 2002. Natural genetic recombination between co-circulating heterotypic enteroviruses. J Gen Virol 83, 2193-200.

Palacios, G. and Oberste, M.S., 2005. Enteroviruses as agents of emerging infectious diseases. J Neurovirol 11, 424-33.

Pallansch, M. and Roos, R. 2001. Enteroviruses: polioviruses, coxsackieviruses, echoviruses, and newer enteroviruses. In: D. Knipe and P. Howley (Eds), Fields Virology, Vol. 1, Lippincott Williams and Wilkins, Philadelphia, pp. 723-775.

Rakoto-Andrianarivelo, M., Guillot, S., Iber, J., Balanant, J., Blondel, B., Riquet, F., Martin, J., Kew, O., Randriamanalina, B., Razafinimpiasa, L., Rousset, D. and Delpeyroux, F., 2007. Co-Circulation and Evolution of Polioviruses and Species C Enteroviruses in a District of Madagascar. PLoS Pathog 3, e191.

Rossolini, G.M., Cresti, S., Ingianni, A., Cattani, P., Riccio, M.L. and Satta, G., 1994. Use of deoxyinosine-containing primers vs degenerate primers for polymerase chain reaction based on ambiguous sequence information. Mol Cell Probes 8, 91-8.

Rousset, D., Rakoto-Andrianarivelo, M., Razafindratsimandresy, R., Randriamanalina, B., Guillot, S., Balanant, J., Mauclere, P. and Delpeyroux, F., 2003. Recombinant vaccine-derived poliovirus in Madagascar. Emerg Infect Dis 9, 885-7.

Santti, J., Hyypia, T., Kinnunen, L. and Salminen, M., 1999. Evidence of recombination among enteroviruses. J Virol 73, 8741-9.

Simmonds, P. and Welch, J., 2006. Frequency and dynamics of recombination within different species of human enteroviruses. J Virol 80, 483-93.

Thoelen, I., Lemey, P., Van Der Donck, I., Beuselinck, K., Lindberg, A.M. and Van Ranst, M., 2003. Molecular typing and epidemiology of enteroviruses identified from an 
outbreak of aseptic meningitis in Belgium during the summer of 2000. J Med Virol 70, $420-9$. 


\section{Figure captions}

Figure 1. Schematic representation of the locations of the primers used in the RT-PCR and RT-snPCR. (A) Scheme of the organization of the human enterovirus genomic RNA. Boxes represent the structural and non-structural protein-encoding sequences of the unique ORF. 5'- and 3'-UTR are represented by lines. (B) Amplicons generated by RT-PCR and SnPCR.

Figure 2. Sensitivity of the different RT-PCR and RT-snPCR on PV-1 RNA extracted from 4-fold serial dilutions. The same DNA ladder was used on each gel; the scale, expressed in $\mathrm{bp}$, is indicated on the top left gel. 
(A)

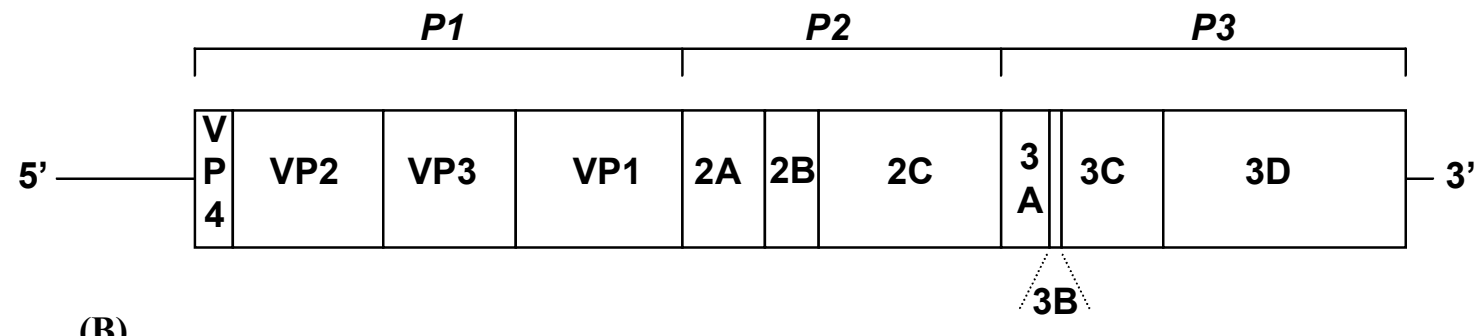

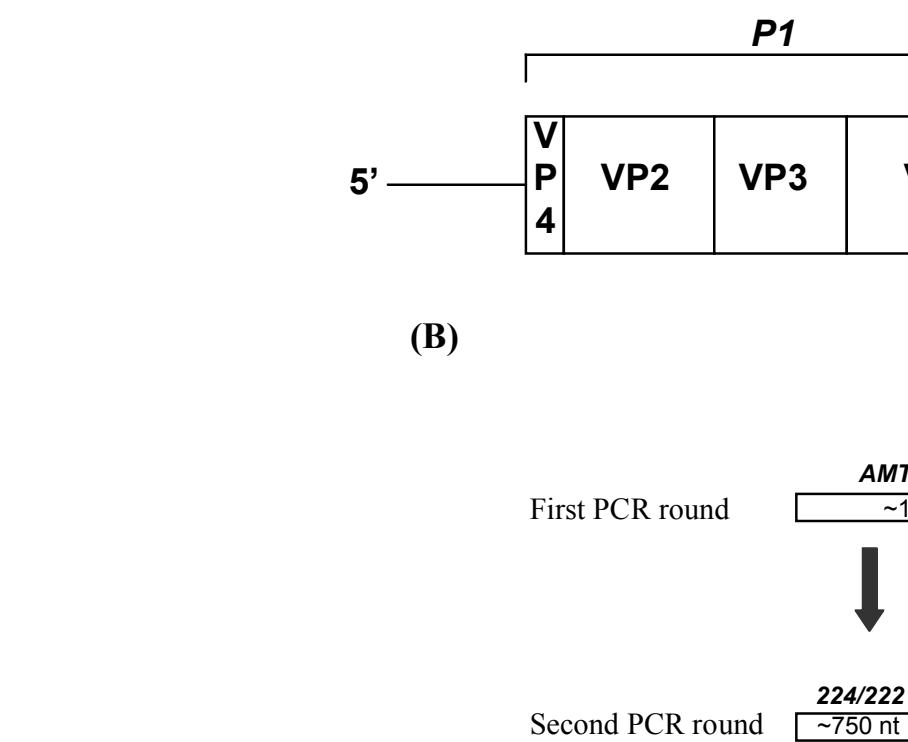

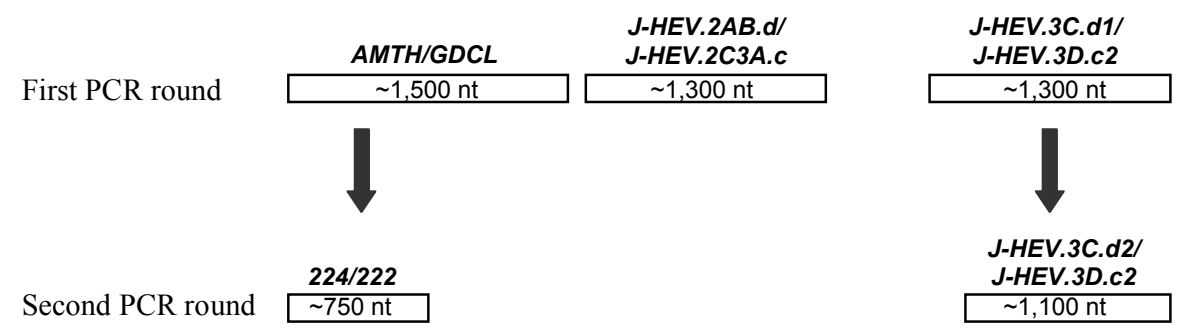

$\sim 1,100 \mathrm{nt}$

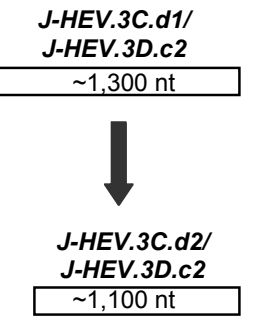

.

Second PCR round $\sim 750 \mathrm{nt}$

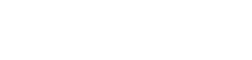

3B 
Dilution factor

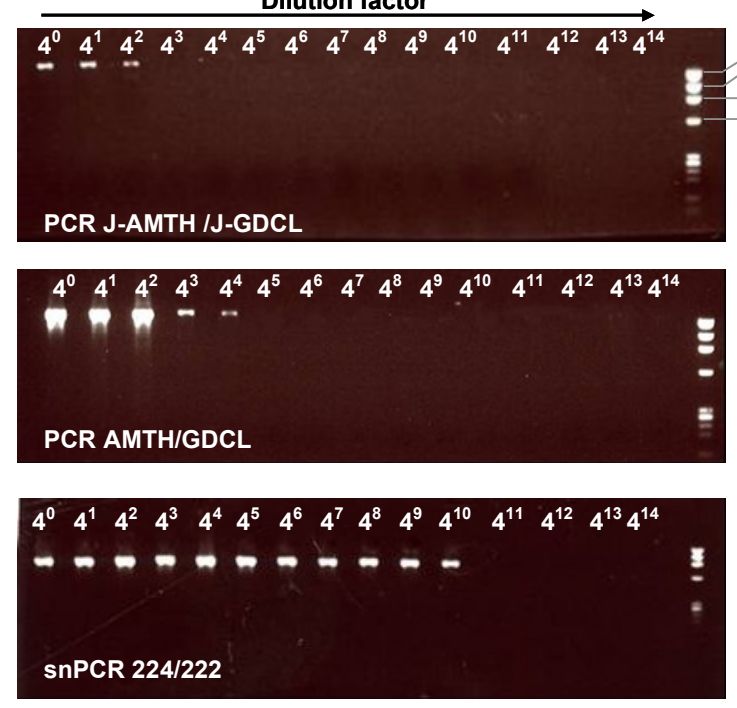

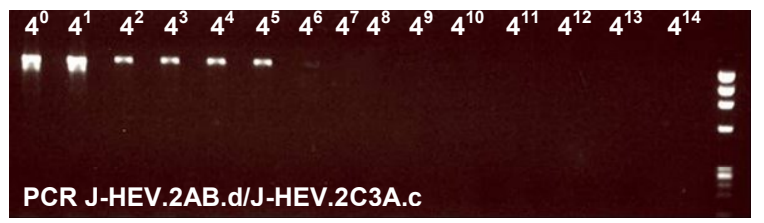

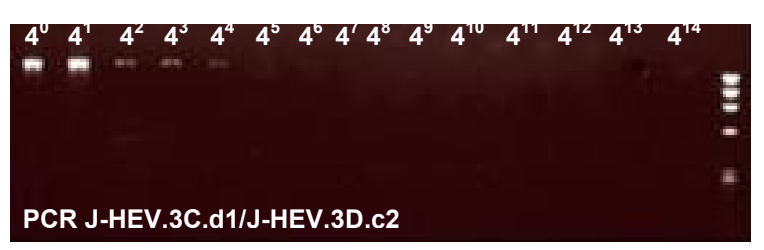

$4^{0} 4^{1} 4^{2} 4^{3} \quad 4^{4} 4^{5} 4^{6} 4^{7} 4^{8} 4^{9} 4^{10} 4^{11} 4^{12} 4^{13} 4^{14}$

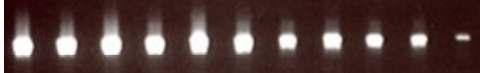

SnPCR J-HEV.3C.d2/J-HEV.3D.c2

Figure(s) 
Table 1. Primers used in this study.

\begin{tabular}{|c|c|c|c|}
\hline Name & $5^{\prime} \rightarrow 3^{\prime}$ sequence $^{\text {a }}$ & Amino-acid motif & Genome position $^{b}$ \\
\hline J-AMTH & GTAAAACGACGGCCAGGCIATGYTIGSIACICAYVT & AML(G/A)TH(V/I/L/M) & $2204-2223$ \\
\hline AMTH & GCIATGYTIGSIACICAYVT & $\operatorname{AML}(\mathrm{G} / \mathrm{A}) \mathrm{TH}(\mathrm{V} / \mathrm{I} / \mathrm{L} / \mathrm{M})$ & $2204-2223$ \\
\hline J-GDCL & CAGGAAACAGCTATGACARNABNCCNCCRCARTCNCC & GDCGG(I/V)L & $3714-3695$ \\
\hline GDCL & ARNABNCCNCCRCARTCNCC & GDCGG(I/V)L & $3714-3695$ \\
\hline $224^{\mathrm{c}}$ & GCIATGYTIGGIACICAYRT & $\operatorname{AMLGTH}(\mathrm{I} / \mathrm{L} / \mathrm{M})$ & $2204-2223$ \\
\hline $222^{\mathrm{c}}$ & CNCCNGGNGGNAYRWACAT & $\mathrm{M}(\mathrm{F} / \mathrm{Y})(\mathrm{I} / \mathrm{V}) \mathrm{PPG}(\mathrm{A} / \mathrm{G})$ & $2960-2942$ \\
\hline J-HEV.2AB.d & GTAAAACGACGGCCAGGAIGYIATGGARCARGG & $(\mathrm{D} / \mathrm{E})(\mathrm{A} / \mathrm{V}) \mathrm{MEQG}$ & $3809-3825$ \\
\hline HEV.2AB.d & GAIGYIATGGARCARGG & $(\mathrm{D} / \mathrm{E})(\mathrm{A} / \mathrm{V}) \mathrm{MEQG}$ & $3809-3825$ \\
\hline J-HEV.2C3A.c & CAGGAAACAGCTATGACGGICCYTGRAAIARIGCYTC & EALFQGP & $5106-5087$ \\
\hline HEV.2C3A.c & GGICCYTGRAAIARIGCYTC & EALFQGP & $5106-5087$ \\
\hline J-HEV.3C.d1 & GTAAAACGACGGCCAGAAYGARAARTTYMGIGAYAT & NEKFRDI & $5666-5685$ \\
\hline HEV.3C.d1 & AAYGARAARTTYMGIGAYAT & NEKFRDI & $5666-5685$ \\
\hline J-HEV.3C.d2 & GTAAAACGACGGCCAGGCIGGICARTGYGGIGGIRT & AGQCGG(V/I) & $5858-5877$ \\
\hline
\end{tabular}




\begin{tabular}{|c|c|c|c|}
\hline J-HEV.3D.c2 & CAGGAAACAGCTATGACACRTCRTCICCRTAIGCIAYCAT & $\mathrm{M}(\mathrm{I} / \mathrm{V}) \mathrm{AYGDDV}$ & $6966-6944$ \\
\hline HEV.3D.c2 & ACRTCRTCICCRTAIGCIAYCAT & $\mathrm{M}(\mathrm{I} / \mathrm{V}) \mathrm{AYGDDV}$ & $6966-6944$ \\
\hline M 13Fwd & GTAAAACGACGGCCAG & & \\
\hline M 13Rev & CAGGAAACAGCTATGAC & & \\
\hline heptaN & NNNNNNN & & \\
\hline
\end{tabular}

a in tailed primers, whose names begin by "J-“, the M13 tags are indicated in italic.

${ }^{\mathrm{b}}$ relative to PV-3 strain Leon.

${ }^{\mathrm{c}}$ according to Nix et al., 2006. 
Table 2. Sensitivity of the RT-PCR and RT-snPCR.

\begin{tabular}{|c|c|c|c|}
\hline $\begin{array}{l}\text { Targeted region of } \\
\text { the viral genome }\end{array}$ & Assay & Primer pair & $\begin{array}{c}\text { Sensitivity }^{\mathbf{a}} \\
\left(\mathrm{TCID}_{50} \text { per } \mathrm{mL}\right)\end{array}$ \\
\hline & PCR & J-AMTH/J-GDCL & $10^{7.2}$ \\
\hline P1 & PCR & AMTH/GDCL & $10^{6.0}$ \\
\hline & snPCR & $224 / 222$ & $10^{3.0}$ \\
\hline $\mathbf{P 2}$ & PCR & $\begin{array}{l}\text { J-HEV.2AB.d/ } \\
\text { J-HEV.2C3A.c } \\
\text { HEV.2AB.d/ } \\
\text { HEV.2C3A.c }\end{array}$ & $10^{5.4}$ \\
\hline P3 & $\begin{array}{l}\text { PCR } \\
\text { PCR } \\
\text { snPCR }\end{array}$ & $\begin{array}{c}\text { J-HEV.3C.d1/ } \\
\text { J-HEV.3D.c2 } \\
\text { HEV.3C.d1/ } \\
\text { HEV.3D.c2 } \\
\text { J-HEV.3C.d2/ } \\
\text { J-HEV.3D.c2 }\end{array}$ & $\begin{array}{l}10^{6.0} \\
10^{5.4} \\
10^{3.0}\end{array}$ \\
\hline
\end{tabular}

${ }^{a}$ For each primer pair, the sensitivity was defined as the titer of the most diluted supernatant allowing the generation of an amplicon. 


\begin{tabular}{|c|c|c|c|}
\hline Serotype & $\begin{array}{c}\text { Number of } \\
\text { isolates }\end{array}$ & $\begin{array}{c}\text { P1 and P3 } \\
\text { genomic parts: } \\
\text { number of } \\
\text { isolates } \\
\text { efficiently } \\
\text { amplified by } \\
\text { PCR without the } \\
\text { need for snPCR }\end{array}$ & $\begin{array}{l}\text { P2 genomic part: } \\
\text { number of } \\
\text { isolates efficiently } \\
\text { amplified by PCR }\end{array}$ \\
\hline HEV-A & & & \\
\hline CV-A2 strain Fleetwood & 1 & 1 & 1 \\
\hline CV-A3 strain Olson & 1 & 1 & 1 \\
\hline CV-A6 strain Gdula & 1 & 1 & 1 \\
\hline CV-A $10 *$ & 2 & 2 & 2 \\
\hline CV-A10 strain Kowalik & 1 & 1 & 1 \\
\hline CV-A12 strain Texas-12 & 1 & 1 & 1 \\
\hline CV-A14 strain G-14 & 1 & 1 & 1 \\
\hline CV-A16 strain G-10 & 1 & 1 & 1 \\
\hline $\mathrm{HEV}-71 *$ & 1 & 1 & 1 \\
\hline \multicolumn{4}{|l|}{ HEV-B } \\
\hline CV-A9* & 1 & 1 & 1 \\
\hline $\mathrm{CV}-\mathrm{B} 1 *$ & 1 & 1 & 1 \\
\hline CV-B4* & 2 & 1 & 1 \\
\hline $\mathrm{CV}-\mathrm{B} 5 *$ & 1 & 1 & 1 \\
\hline $\mathrm{EV}-1^{*}$ & 1 & 1 & 1 \\
\hline
\end{tabular}




\begin{tabular}{|c|c|c|c|}
\hline EV-3* & 4 & 3 & 3 \\
\hline EV-6* & 4 & 1 & 2 \\
\hline EV-7* & 6 & 3 & 3 \\
\hline EV-7 strain Wallace & 1 & 1 & 1 \\
\hline $\mathrm{EV}-8^{*}$ & 2 & 1 & 1 \\
\hline EV-9* & 1 & 1 & 1 \\
\hline $\mathrm{EV}-11 *$ & 4 & 1 & 2 \\
\hline $\mathrm{EV}-12^{*}$ & 3 & 1 & 1 \\
\hline EV-13* & 4 & 3 & 3 \\
\hline EV-19* & 3 & 1 & 1 \\
\hline EV-20* & 3 & 1 & 1 \\
\hline $\mathrm{EV}-21^{*}$ & 3 & 3 & 3 \\
\hline EV-29* & 2 & 1 & 1 \\
\hline EV-30* & 1 & 1 & 1 \\
\hline EV-33* & 2 & 1 & 1 \\
\hline HEV-69* & 1 & 1 & 1 \\
\hline HEV-74* & 2 & 2 & 1 \\
\hline \multicolumn{4}{|l|}{ HEV-C } \\
\hline CV-A11* & 3 & 3 & 3 \\
\hline CV-A13* & 8 & 5 & 6 \\
\hline CV-A17* & 2 & 2 & 2 \\
\hline CV-A20* & 4 & 2 & 2 \\
\hline CV-A21* & 2 & 1 & 1 \\
\hline CV-A24* & 4 & 3 & 4 \\
\hline
\end{tabular}


HEV-D

2

2

2

PV

\begin{tabular}{lccc} 
PV-1 strain Sabin & 1 & 1 & 1 \\
PV-2 strain Sabin & 1 & 1 & 1 \\
PV-3 strain Sabin & 1 & 1 & 1 \\
\hline Total & 90 & 62 & 65
\end{tabular}

Table 3. HEV strains tested for amplification. Prototype viruses are listed with their names.

Asterisks indicate unpublished field strains.

${ }^{a}$ All the isolates which have not been amplified efficiently by PCR in the P1 and P3 parts of their genome were amplified successfully by snPCR. 